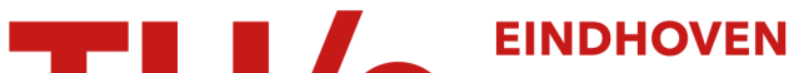 \\ UNIVERSITY OF \\ TECHNOLOGY
}

\section{On linear complementarity systems}

Citation for published version (APA):

Leenaerts, D. M. W. (1999). On linear complementarity systems. IEEE Transactions on Circuits and Systems. I, Fundamental Theory and Applications, 46, 1022-1026. https://doi.org/10.1109/81.780383

DOI:

10.1109/81.780383

Document status and date:

Published: 01/01/1999

\section{Document Version:}

Publisher's PDF, also known as Version of Record (includes final page, issue and volume numbers)

\section{Please check the document version of this publication:}

- A submitted manuscript is the version of the article upon submission and before peer-review. There can be important differences between the submitted version and the official published version of record. People interested in the research are advised to contact the author for the final version of the publication, or visit the $\mathrm{DOI}$ to the publisher's website.

- The final author version and the galley proof are versions of the publication after peer review.

- The final published version features the final layout of the paper including the volume, issue and page numbers.

Link to publication

\section{General rights}

Copyright and moral rights for the publications made accessible in the public portal are retained by the authors and/or other copyright owners and it is a condition of accessing publications that users recognise and abide by the legal requirements associated with these rights.

- Users may download and print one copy of any publication from the public portal for the purpose of private study or research.

- You may not further distribute the material or use it for any profit-making activity or commercial gain

- You may freely distribute the URL identifying the publication in the public portal.

If the publication is distributed under the terms of Article $25 \mathrm{fa}$ of the Dutch Copyright Act, indicated by the "Taverne" license above, please follow below link for the End User Agreement:

www.tue.nl/taverne

Take down policy

If you believe that this document breaches copyright please contact us at:

openaccess@tue.nl

providing details and we will investigate your claim. 


\section{REFERENCES}

[1] H. Fukuy, "The noise performance of microwave transistors," IEEE Trans. Electron Devices, vol. ED-13, pp. 329-341, Mar. 1966.

[2] H. Hillbrand and P. H. Russer, "An efficient method for computer aided noise analysis of linear amplifier networks," IEEE Trans. Circuits Syst., vol. CAS-23, pp. 235-238, Apr. 1976.

[3] V. Rizzoli and A. Lipparini, "Computer-aided noise analysis of linear multiport networks of arbitrary topology," IEEE Trans. Microwave Theory Tech., vol. MTT-33, pp. 1507-1512, Dec. 1985.

[4] J. A. Dobrowolski, "A CAD-oriented method for noise figure computation of two-ports with any internal topology," IEEE Trans. Microwave Theory Tech., vol. 37, pp. 15-20, Jan. 1989.

[5] M. E. Mokari and W. Patience, "A new method of noise parameter calculation using direct matrix analysis," IEEE Trans. Circuits Syst., vol. CAS-39, pp. 767-771, Sept. 1992.

[6] J. A. Dobrowolski, Introduction to Computer Methods for Microwave Circuit Analysis and Design. Norwood, MA: Artech, 1991.

[7] M. Reed and B. Simon, Methods of Modern Mathematical Physics. London, U.K.: Academic, 1972.

[8] J. Lange, "Noise characterization of linear two-ports in terms of invariant parameters," IEEE J. Solid-State Circuits, vol. SC-2, pp. 37-40, June 1967.

[9] R. J. Hawkins, "Limitations of Nielsen's and related noise equations applied to microwave bipolar transistors, and a new expression for the frequency and current dependent noise figure," Solid State Electron., Vol. 20, pp. 191-196, 1977.

[10] R. A. Pucel and U. L. Rhode, "An exact expression for the noise resistance for the Hawkins bipolar noise model," IEEE Microwave Guided Wave Lett., vol. 3, pp. 35-37, Feb. 1993.

\section{On Linear Dynamic Complementary Systems}

\author{
Domine M. W. Leenaerts
}

\begin{abstract}
A new method will be proposed to solve dynamic linear complementary systems. To this class of systems belong all electrical piecewise linear (PL) dynamic networks. The method uses numerical integration methods to transform the original problem into a static standard linear complementary problem (LCP) that needs to be solved for each time step period. The method deals efficiently with inconsistent initial conditions and finds implicitly which mode changes and how the system equations are influenced by this change.
\end{abstract}

Index Terms-Circuit analysis, linear complementary problem, linear dynamic complementary problem, piecewise linear systems.

\section{INTRODUCTION}

Many electrical networks consist of resistors, ideal diodes, and nonlinear elements such as, e.g., transistors. To analyze the behavior of such networks, the behavior of the nonlinear elements is often piecewise linear (PL) approximated. In the literature, many papers can be found dealing with possible model descriptions to store the PL data information in a minimum way [1]-[3]. In addition, much research is done in the field of analyzing networks with PL components [4], [5].

It is known that PL model descriptions are related to the linear complementary problem (LCP) [4], [6]. This problem is widely

Manuscript received February 26, 1998; revised September 18, 1998. This paper was recommended by Associate Editor J. Vlach.

The author is with the Department of Electrical Engineering, Technical University Eindhoven, 5600MB Eindhoven, The Netherlands.

Publisher Item Identifier S 1057-7122(99)06356-4.

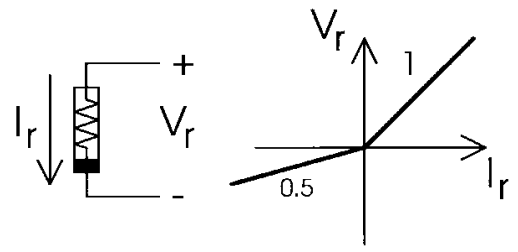

Fig. 1. Nonlinear resistor with PL characteristic.

studied in mathematical science [7]. Recently, it has been shown that the dynamics in systems with complementary conditions can be described as a linear dynamic complementary problem (LDCP) [8], [9]. Electrical networks with PL components and dynamic elements such as capacitors and inductors belong to this problem. In [8] and [9] a possible solution technique was proposed, based on the rational Laplace transformation. However, from an analysis point of view, this method is not suitable for implementation in a simulator.

The purpose of the research is to show that the LDCP can be treated as a sequence of LCP's that must be solved for each time step. Consequently, the LDCP is solved faster than [9] and electrical networks with PL components can be solved using the standard techniques as, for example, the one discussed in [5]. Furthermore, it will be shown that the proposed method can deal efficiently with inconsistent initial conditions; a problem which occurs in networks with ideal switches and diodes. The proposed method implicitly detects which switch or diode is changing and obtains the correct system equations after the switching instant. This is an improvement on [13] where the system equations need to be reformulated and where it is not explained how to detect that the switch is changing. Also, in [14] additional steps have to be taken in order to find the state equations after switching.

To highlight the basic problem, the relation between PL and LCP will be discussed in Section II. Then the LDCP will be treated in Section III. Next, the proposed method will be discussed and demonstrated by an example. Inconsistent initial conditions will be discussed in Section VI. Some concluding remarks are given in Section VII.

\section{Relation Between PL Modeling And LCP}

In PL electronic networks, the nonlinear behavior of component elements is PL approximated. Consider as an example the fairly simple nonlinear resistor in Fig. 1, having a two-segment PL characteristic. The two segments are described by

$$
\begin{aligned}
& V_{r}=I_{r}, \quad I_{r} \geq 0 \\
& V_{r}=\frac{1}{2} I_{r}, \quad I_{r}<0 .
\end{aligned}
$$

This equation can be formalized into the state model description [4]

$$
\begin{gathered}
V_{r}=\frac{1}{2} I_{r}+\left(\frac{1}{2}\right) u, \quad j=-\frac{1}{2} I_{r}+\frac{1}{2} u \\
u \geq 0, j \geq 0, u^{T} j=0
\end{gathered}
$$

with internal state variables $u$ and $j$. As long as $I_{r}$ remains positive, it follows that $u \geq 0, j=0$ and the impedance is $1 \Omega$. If $I_{r}$ becomes negative, one obtains $u=0, j \geq 0$ and the port impedance becomes $\frac{1}{2} \Omega$. Equation (3) is known as the complementary condition and reflects two modes: $u=0, j \geq 0$ and $u \geq 0, j=0$. In general, $k$ breakpoints in a PL characteristic represent $2^{k}$ modes. In addition, other model descriptions exist, e.g., the models of Chua, Kahlert, and 


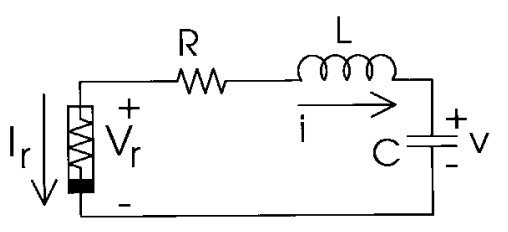

Fig. 2. Simple dynamic electrical network with one PL element. The parameter settings are $L=1 \mathrm{H}, C=1 \mathrm{~F}$, and $R=1 \Omega$.

Leenaerts [1]-[3]. In [6] it has been shown that all model descriptions are a special case of the state model description. In other words, the state model description is the most general model known to model any PL function. Almost any electrical component behavior that is continuous can be modeled using PL techniques, including transistor networks [10].

The system described by the second equation in (2) together with (3) is a special case of the $k$-dimensional system

$$
\begin{aligned}
j & =M u+q \quad u \geq 0, j \geq 0 \\
u^{T} j & =0
\end{aligned}
$$

with matrix $M \in R^{k \times k}$ and $q \in R^{k}$. To find $u, j \in R^{k}$ consistent with (4) for a given $q$ is a well-known problem in mathematical programming, called the LCP [7]. Several solution strategies are developed to solve the LCP such as, for instance, the method proposed by Katzenelson and Lemke [7], [11]. Note that although Katzenelson's algorithm is developed to solve networks with PL representations of the nonlinearities, implicitly, the method solves an LCP. Katzenelson's algorithm traces a path in the solution space, starting with an input $q^{*}$ for which the solution is known. The input $q^{*}$ is now linearly modified to the input $q$ for which the problem should be solved using a parameter $\lambda$. This results in the process

$$
j=M u+q^{*}+\lambda\left(q-q^{*}\right), \quad 0 \leq \lambda \leq 1
$$

terminating for $\lambda=1$ and yielding the correct solution for $u$ and $j$. Note that many PL network simulators use the Katzenelson algorithm to analyze the network and, consequently, use Katzenelson to solve the underlying LCP [5].

\section{Linear Dynamic Complementary Problem}

So far we have only discussed PL networks having static components. The question arises as to what happens to the LCP if dynamic component elements such as capacitors are included in the PL network. As an example, consider Fig. 2 where PL resistor (1) is used. Using state variables $v$ and $i$ the network is described by

$$
\dot{v}=\frac{1}{C} i, \quad i=\frac{1}{L}\left(-v-R i+V_{r}\right) .
$$

Using (2) to remove variable $V_{r}$, the network dynamics are finally expressed as

$$
\begin{aligned}
& \dot{x}(t)=\left(\begin{array}{cc}
0 & \frac{1}{C} \\
-\frac{1}{L} & -\frac{R}{L}-\frac{1}{2 L}
\end{array}\right) x(t)+\left(\begin{array}{c}
0 \\
-\frac{1}{2 L}
\end{array}\right) u(t) \\
& y(t)=\left(\begin{array}{ll}
0 & -\frac{1}{2}
\end{array}\right) x(t)+\left(\frac{1}{2}\right) u(t) \\
& u \geq 0, y \geq 0, u^{T} y=0
\end{aligned}
$$

with $x_{1}=v, x_{2}=i$, and $y=j$. The vectors $y(t)$ and $u(t)$ represent the changes of the segments in the PL function. The state vector $x(t)$, together with $y(t)$ and $u(t)$, describe the complete system behavior in a minimum manner. The necessity of $y$ and $u$ is inherent in the fact that any PL network deals with the LCP. Removing $y$ (or $u$ ) from the system equations when becoming zero will not reduce the amount of computation. One can detect when $y$ (or $u$ ) becomes positive (although this might be not trivial in the case where several modes change at the same instant), but the question arises as to how the system equations are changed. For $k$ breakpoints there are $2^{k}$ possible system equations and to retain them all will cost more than to keep $k$ additional equations.

In a more formal way, (7) can be written as

$$
\begin{gathered}
\dot{x}(t)=A x(t)+B u(t), \quad y(t)=C x(t)+D u(t), \\
u \geq 0, y \geq 0, u^{T} y=0
\end{gathered}
$$

with the $n$-dimensional state variable $x$ representing the voltages on capacitors and currents through inductors. The problem of solving (8) under restriction of complementary condition (3) is known as the LDCP [8]. In the LDCP, functions $u(\cdot), x(\cdot)$, and $y(\cdot)$ take values in $R^{k}, R^{n}$ and $R^{k}$, respectively and $A, B, C$, and $D$ are constant matrices of appropriate dimensions. Due to the complementary condition for $y$ and $u$, the system has $2^{k}$ modes, each represented by a set of linear ordinary differential equations (ODE's). These ODE's can be solved using numerical integration methods.

In [8] and [9] a method is presented to solve the LDCP based on the rational Laplace transformation. In this method, the numerical integration is stopped at the moment the complementary conditions are violated. The system then must be transformed to the Laplace domain and several additional computations have to be performed. To avoid this break and these additional steps, a new method will be proposed.

\section{The Proposed Method}

In conventional network simulators numerical integration methods are used to solve time-dependent equations of network components [12]. Applying an integration method to a dynamic electrical network component results in a sequence of small static replacement circuits. The component values in this replacement circuit are determined by the used integration method, the step size, and the history of integration.

The main idea is now to rewrite the LDCP (8) as a sequence of static LCP's, similar to (4). The entries in the matrix $M$ and vector $q$ are then determined by the original problem and the integration method. The solution of the static LCP at a certain time instance can be obtained by applying existing methods, e.g., Katzenelson's algorithm. After obtaining this solution a new time step may be performed, after which a new static LCP is obtained. In this way, the procedure may continue until the final time of integration is reached.

To this purpose consider the well-known trapezoidal integration method [12]

$$
z_{n+1}=\frac{h}{2}\left(\dot{z}_{n+1}+\dot{z}_{n}\right)+z_{n}=P_{n}+\beta_{-1} \dot{z}_{n+1}
$$

(or the backward Euler formula $z_{n+1}=z_{n}+h \dot{z}_{n+1}$ ). Here, $z_{n}$ represents the computed solution at time $t_{n}$ and $h_{n}$ is the time step at time $t_{n}$. Expression (9) integrates over the time period $t_{n} \rightarrow t_{n+1}$. For the next time step, $P_{n+1}$ is needed and for the trapezoidal rule the update from $P_{n}$ into $P_{n+1}$ can be found as $P_{n+1}=-P_{n}+2 z_{n+1}$ (in the case of backward Euler $P_{n+1}=z_{n+1}$ ).

Applying the numerical integration method (9) to (8) yields

$$
\begin{aligned}
\beta_{-1}^{-1}\left(x_{n+1}-P_{n}\right) & =A x_{n+1}+B u_{n+1} \\
y_{n+1} & =C x_{n+1}+D u_{n+1}
\end{aligned}
$$

and, thus,

$$
x_{n+1}=\left(\beta_{-1}^{-1} I-A\right)^{-1}\left[B u_{n+1}+\beta_{-1}^{-1} P_{n}\right]
$$




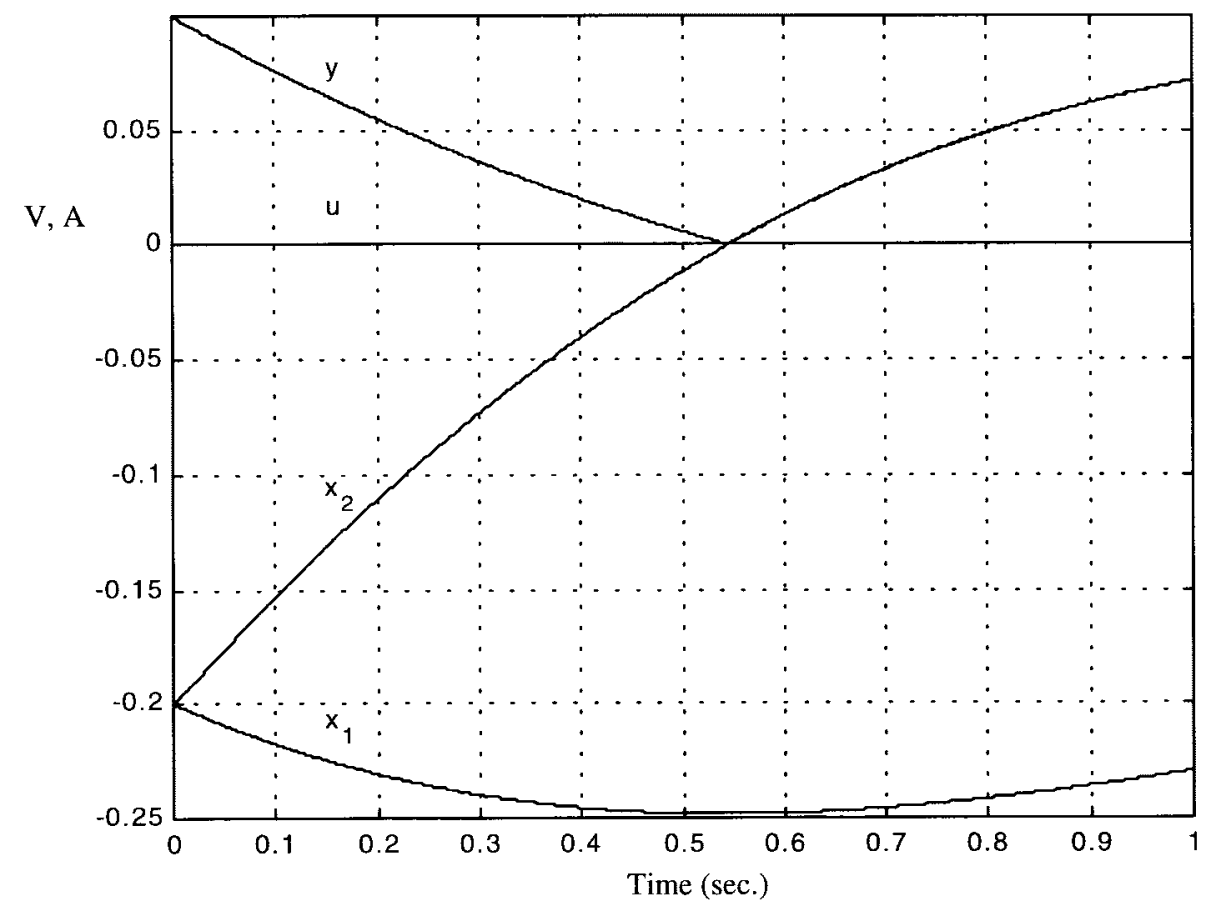

Fig. 3. Solutions of the dynamic network of Fig. 2.

valid within $t_{n} \rightarrow t_{n+1}=t_{n}+h$. One can now use (11) to reformulate the second equation in (10) as a standard LCP, yielding

$$
\begin{aligned}
y_{n+1}= & \left(C\left(\beta_{-1}^{-1} I-A\right)^{-1} B+D\right) u_{n+1} \\
& +C \beta_{-1}^{-1}\left(\beta_{-1}^{-1} I-A\right)^{-1} P_{n}, \\
& \quad y_{n+1} \geq 0, u_{n+1} \geq 0, y_{n+1}^{T} u_{n+1}=0
\end{aligned}
$$

or

$$
\begin{aligned}
& y_{n+1}=M_{n+1} u_{n+1}+q_{n+1} \\
& y_{n+1} \geq 0, u_{n+1} \geq 0, y_{n+1}^{T} u_{n+1}=0 .
\end{aligned}
$$

The obtained result (13) means that the dynamic LDCP can be solved as a sequence of static standard LCP's: one for each time period. The LCP reflects the nonlinear behavior of the network in terms of PL elements.

In time period $t_{n} \rightarrow t_{n+1}$, the static LCP (13) must be solved. After obtaining the solution in terms of $u_{n+1}$ and $y_{n+1}$, the next time period can be started, i.e., $t_{n+1} \rightarrow t_{n+2}$. Consider the time period $t_{n} \rightarrow t_{n+1}$ and assume that $P_{n}$ is known. Two situations need to be considered.

\section{A. The Case If $q_{n+1} \geq 0$ for All Entries}

Knowing step size $h$ and $P_{n}$, LCP (13) is completely determined. The solution of the LCP in this case is readily obtained as $y_{n+1}=$ $q_{n+1}, u_{n+1}=0$. The next step is to calculate $x_{n+1}$ from (11). To solve the problem in the next time frame $t_{n+1} \rightarrow t_{n+2}, P_{n+1}, M_{n+2}$ and $q_{n+2}$ can be obtained from $x_{n+1}$ and (12), respectively.

\section{B. The Case If Some Entries of $q_{n+1}$ Are Negative}

A new mode is entered in the case where one or more entries of $q_{n+1}$ are negative. First, the LCP (13) must be solved to find which mode is entered. Because one or more modes change, some of the entries in $u_{n+1}$ become positive and the corresponding entries in $y_{n+1}$ become zero in order to be consistent with the complementary conditions. In the next step, $x_{n+1}$ is calculated from (11). Again, as

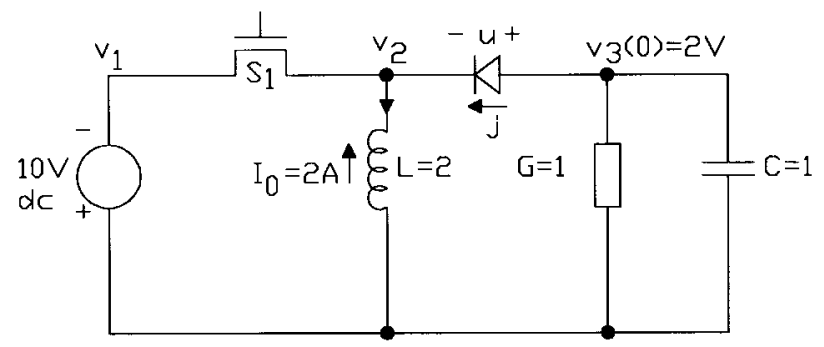

Fig. 4. Switching network with inconsistent initial conditions, as taken from [13]. In the network an ideal PL diode is used, while in [13] an ideal network diode was used. In order to have the same results, the diode is differently situated in this work, as compared to [13]

the next step $P_{n+1}, M_{n+2}$ and $q_{n+2}$ need to be calculated and one may proceed with the next time instant. However, because a mode is changed, inconsistent initial conditions can occur. This issue will be treated in Section VI.

\section{Some Remarks}

Equation (12) calls for the inversion of $\left(\beta_{-1}^{-1} I-A\right)$. For fixed step-size numerical integration algorithms $\beta_{-1}^{-1}$ is fixed and the inversion needs to be determined only once. However, if variable step size is used, for each new time step $h$ the inversion needs to be recomputed. First, note that matrix $A$ is updated over its diagonal by the step size. Second, if one writes $G=\left(\beta_{-1}^{-1} I-A\right)^{-1} B=$ $\left(g_{1}, g_{2}, \cdots, g_{k}\right)$ it can be observed that the columns $g_{1}$ through $g_{k}$ can be found by solving the systems $\left(\beta_{-1}^{-1} I-A\right) g_{i}=b_{i}$ for $i=1, \cdots, k$. Both properties allow for an efficient computation of the inverse matrix [15]. Note also that $\left(\beta_{-1}^{-1} I-A\right)$ is not influenced by the change of a mode and need not be recomputed during the solution process of the static LCP.

To conclude, the detection of an event, the change of mode, and the continuation of the integration are within the same routine. Only a standard LCP has to be solved but, within PL simulators, tools for doing this are already available. 


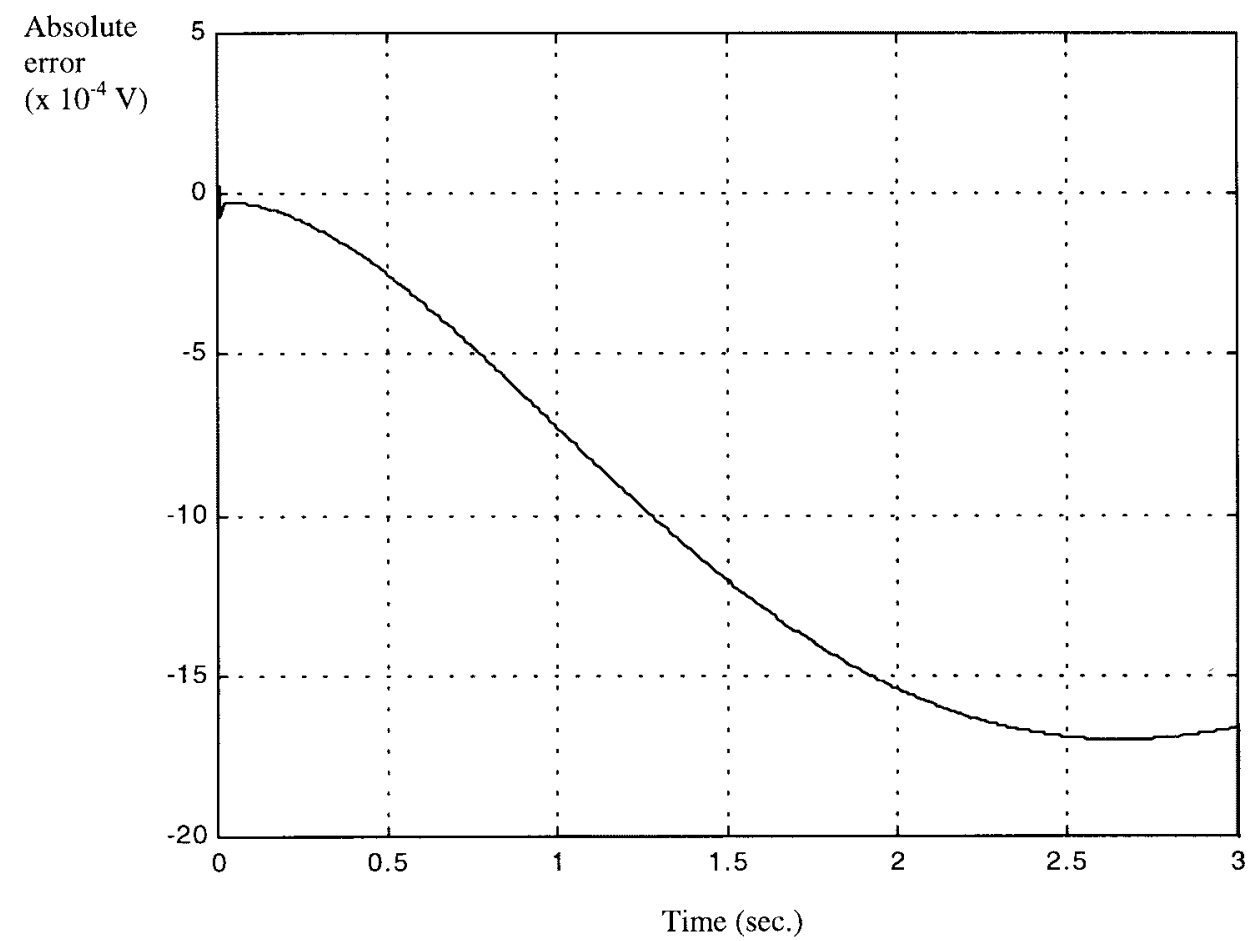

Fig. 5. The absolute error between the simulated and the exact solution of the network in Fig. 4.

\section{EXAMPLE}

Consider again the electrical network of Fig. 2. Assume $R=$ $L=C=1$ and that the initial condition is set to $x(0)=x_{0}=$ $(-0.2,-0.2)^{T}$. The fixed step size $h$ is chosen equal to $h=0.001$ s. The trapezoidal rule will be used, yielding $P_{n+1}=-P_{n}+2 x_{n+1}$. For $M_{n+1}=M$ we obtain $M=0.5001$ while $q_{0}=0.0999$. Consequently, the LCP $j_{0}=M u_{0}+q_{0}$ has the solution $u_{0}=0, j_{0}=$ $y_{0}=q_{0}$ and the nonlinear resistor has the resistance of $\frac{1}{2} \Omega$. At time instance $t_{n}=0.546+h$, we obtain $P_{n}=(-0.2484,-0.0003)^{T}$, $x_{n}=(-0.2484,0)^{T}$, and $q_{n+1}=-2.0381 e^{-5}$. The LCP yields $j_{n+1}=0.5001 u_{n+1}-2.0381 e^{-5}$ and, therefore, a change in mode is detected. The resistance becomes $1 \Omega$ at $x_{n, 2}=i=0$. After solving the LCP, we have $u_{n+1}=\left(-q_{n+1}\right) / M=4.0751 e^{-5}, j_{n+1}=0$. To proceed, $P_{n+1}$ follows from $x_{n+1}$ and to compute the new $x_{n+1}$ after the change in mode, (11) is used with $u_{n+1}=4.0751 e^{-5}$, giving $x_{n+1}=(-0.2484,0)^{T}$. Therefore, the state variable values before the mode changes are the same as after the change. The conclusion is that no inconsistent initial condition occurred during the transition and the method may proceed without modification. The resulting dynamic behavior of the circuit can be seen in Fig. 3. The proposed method as well as [9] have been implemented in MATLAB to simulate the example. The simulation with the new method took $1.45 \mathrm{CPU} \mathrm{s}$, compared to 3.21 CPU s for the method in [9]. Using the specific PL simulator PLANET [16], the problem was solved within 2.01 CPU $\mathrm{s}$. The results show the improvement in computation time obtained with the new approach.

\section{INCONSISTENT INITIAL CONDITIONS}

From the example, the question may arise as to what happens if the state variable values before and after a mode changes are not the same. Then the network has inconsistent initial conditions. As an example, take the circuit from [13] wherea solution method is also provided (see Fig. 4). As in [13], the switch opens when the inductor current is $2 \mathrm{~A}$, and at this instant the initial voltage on the capacitor is $2 \mathrm{~V}$. The system equations are then given as

$$
\begin{aligned}
{\left[\begin{array}{c}
\dot{v}_{3} \\
\dot{i}
\end{array}\right] } & =\left[\begin{array}{rr}
-\frac{G}{C} & -\frac{1}{C} \\
\frac{1}{L} & 0
\end{array}\right]\left[\begin{array}{c}
v_{3} \\
i
\end{array}\right]+\left[\begin{array}{l}
0 \\
\frac{1}{L}
\end{array}\right] u, \\
j & =\left[\begin{array}{ll}
0 & 1
\end{array}\right]\left[\begin{array}{c}
v_{3} \\
i
\end{array}\right]+[0] u
\end{aligned}
$$

with the complementary conditions still valid. The initial conditions at the instant of switching are $x_{0}=\left(v_{3}, i\right)^{T}=(2,-2)^{T}$. Due to the voltage $v_{2}=-10 \mathrm{~V}$ before switching, the ideal diode is assumed to be off. The fixed step size is set to $h=0.01$ and the trapezoidal rule will be applied.

For the first time period $t=0+h$ one obtains $M=0.0025$, $P_{0}=x_{0}$ and $q_{1}=-1.995$. At this instant, the LCP needs to be updated, resulting in $u_{1}=798.01$ (note that due to the difference between an ideal network diode and an ideal diode in PL techniques, this result means that the inductor is direct connected to $G$ and $C$ ). Consequently, from (11) $x_{1}=(1.99,0)^{T}$, which is not the same as $x_{0}$, the value before the switching instant. The system deals with inconsistent initial conditions and to overcome the problem, one returns to the original solution vector at switching instant $x_{0}$. The system is considered in the current topology, i.e., the diode is conducting [13]. Therefore, $P_{1}$ is again equal to $x_{1}=x_{0}$. One may now continue and the error between $v_{3}(t)$ and the exact solution $\left(v_{3}(t)=2 e^{-0.5 t}(\cos 0.5 t+\sin 0.5 t) u(t)[13]\right)$ is depicted in Fig. 5 and shows good agreement, even for fairly large $h$.

We conclude that the following holds.

- If a mode changes and the state vectors before and after the instant are equal, we continue the integration.

- If a mode changes and the state vectors before and after the instant are different, we reset the integration method and the state vector obtained before the instant is used as initial condition.

Note that the topology of the network changes due to the change in mode. However, it is not necessary to describe the system equations in the new situation explicitly, as is necessary in [13]. Because (8) 
contains all information of all possible topologies, it is known how the system equations will change due to a change in mode of a diode. Furthermore, the proposed method automatically detects which modes will change, even if this occurs at the same switching instant. The price to pay is to keep one additional variable per ideal diode/switch in the system equations.

\section{CONCLUSION}

We showed that the LDCP can be transformed in a sequence of LCP's. In each time step, a single LCP has to be solved and the used numerical integration method needs to be reset only when the initial conditions are inconsistent. This is simply detected by comparing the state vectors before and after the switching instant. Because the proposed method integrates the LCP and the numerical integration into a single algorithm, the change of mode and the continuation of the integration are done implicitly. This is an improvement over the method in [9]. The method can be used advantageously in (especially PL) simulation environments.

\section{REFERENCES}

[1] S. M. Kang and L. O. Chua, "A global representation of multidimensional piecewise linear functions with linear partitions," IEEE Trans. Circuits Syst., vol. 25, pp. 938-940, Nov. 1978.

[2] C. Kahlert and L. O. Chua, "A generalized canonical piecewise linear representation," IEEE Trans. Circuits Syst., vol. 37, pp. 373-382, Mar. 1990.

[3] D. M. W. Leenaerts, "Further extensions to Chua's explicit piecewise linear function descriptions," Int. J. Circuit Theory Appl., vol. 24, pp. 621-633, 1996.

[4] W. M. G. van Bokhoven, "Piecewise linear analysis and simulation," in Circuit Analysis, Simulation and Design, A. E. Ruehli, Ed. Amsterdam, The Netherlands: North-Holland, 1986, ch. 9.

[5] P. Pejovic and D. Maksimovic, "An algorithm for solving piecewise linear networks that include elements with discontinuous characteristics," IEEE Trans. Circuits and Syst. I, vol. 43, pp. 453-460, 1996.

[6] T. A. M. Kevenaar and D. M. W. Leenaerts, "A comparison of piecewise-linear model descriptions," IEEE Trans. Circuits., Syst. I, vol. 39, pp. 996-1004, Dec. 1992.

[7] R. W. Cottle, J.-S. Pang, and R. E. Stone, The Linear Complementary Problem. Boston, MA: Academic, 1992.

[8] J. M. Schumacher, "Some modeling aspects of unilaterally constrained dynamics," in Proc. ESA Int. Workshop Advanced Mathematical Methods Dynamics of Flexible Bodies, ESA-ESTEC, Noordwijk, The Netherlands, June 1996.

[9] W. P. M. H. Heemels, J. M. Schumacher, and S. Weiland, "Complete description of dynamics in the linear complementary-slackness class of hybrid systems," in Proc. 6th IEEE Conf. Decision Control 1997, San Diego, CA, pp. 1243-1248.

[10] W. Kruiskamp and D. Leenaerts, "Behavioral and macro modeling using piecewise linear techniques," Int. J. Analog Integrated Circuits Signal Processing, vol. 10, nos. 1/2, pp. 67-76, 1996.

[11] J. Katzenelson, "An algorithm for solving nonlinear resistor networks," Bell Syst. Tech. J., vol. 44, pp. 1605-1620, 1965.

[12] J. Vlach and K. Singhal, Computer Methods for Circuit Analysis and Design. New York: Van Nostrand Reinhold, 1983

[13] J. Vlach, J. M. Wojciechowski, and A. Opal, "Analysis of nonlinear networks with inconsistent initial conditions," IEEE Trans. Circuits Syst. I, vol. 42, pp. 195-200, Apr. 1995.

[14] A. Massarini, U. Reggiani, and M. K. Kazimierczuk, "Analysis of networks with ideal switches by state equations," IEEE Trans. Circuits Syst. I, vol. 44, pp. 692-697, Aug. 1997.

[15] B. N. Datta, Numerical Linear Algebra and Applications. Pacific Grove, CA: Brooks/Cole, 1995.

[16] D. M. W. Leenaerts and W. M. G. van Bokhoven, Piecewise Linear Modeling and Analysis. New York: Kluwer, 1998.

\section{A Novel Stability Analysis of a PLL for Timing Recovery in Hard Disk Drives}

\author{
Alessandro De Gloria, Daniele Grosso, \\ Mauro Olivieri, and Giuseppe Restani
}

\begin{abstract}
Timing recovery in hard-disk-drive data transfer is an increasingly critical problem due to the growing data rate to be supported. We present a novel approach to the stability analysis of a high-order digital phase-locked loop (PLL), addressing the specific application. We find the value of the integrating and proportional open-loop gain leading to stable behavior. The analysis was developed in both the digital and analog domains and the obtained results are compared. The stability analysis in the unified transforms domain is also developed, showing that it matches the results obtained with both discrete and continuous-time analysis.
\end{abstract}

Index Terms - Geometric method, stability analysis, timing recovery, unified transforms domain.

\section{INTRODUCTION}

Phase-locked loop (PLL)-based timing recovery [1], [2] is often used in high-speed synchronous communication receivers to adjust the frequency and the phase of the receiver clock to optimize the sampling instants of the received signal. A particularly critical application is timing recovery applied to magnetic recording systems. It has been shown [3]-[5] that a large improvement in terms of storage density and reliability is achieved by introducing techniques, such as timing recovery, adaptive equalization, partial response, and sequence detection. As PLL performance strongly affects the behavior of the overall system, we focused on studying its stability [6]-[9]. We used a geometric method that allowed us to have a quick and precise analysis of the high-order parameterized dynamic system that represents the PLL. Our reference architecture is the PLL with a loop delay of $M$ cycle shown in Fig. 1(a) where the parameters $\alpha$ and $\beta$ are, respectively, the integrating and the proportional open-loop gain. This work was aimed at studying a PLL with $M=13$, but the method we used is valid for any value of the loop delay. Our purpose was to find the values of $\alpha$ and $\beta$ that guarantee the loop stability. We developed the analysis in both discrete time (DT) and continuous time (CT) domains. Furthermore, we compared the achieved results. In addition, we used the delta operator and the unified transform theory [10] that allows one to unify continuous- and discrete-time theory. Referring to Fig. 1(a) and considering $t_{k}$ as the input signal and $I_{k}$ as the output signal, the transfer function of the system is

$$
G(z)=\frac{(z-1) \cdot \beta+\alpha}{(z-1)^{2} \cdot z^{M}+(z-1) \cdot \beta+\alpha}=\frac{I_{k}}{t_{k}} .
$$

\section{Stability Analysis IN the $Z$ Domain}

\section{A. PLL with No Loop Delay}

Assume $M=0$ and consider the denominator of (1) $[F(z)]$. The stability analysis can be realized by applying the bilinear transform

Manuscript received April 18, 1997; revised April 16, 1998. This paper was recommended by Associate Editor O. Feely.

The authors are with DIBE-University of Genoa, Genoa, Italy.

Publisher Item Identifier S 1057-7122(99)06365-5. 